\title{
Work-related risk factors for specific shoulder disorders: a systematic review and meta-analysis
}

\author{
Henk F van der Molen, ${ }^{1,2,3}$ Chiara Foresti, ${ }^{4}$ Joost G Daams, ${ }^{1,2,3}$ \\ Monique H W Frings-Dresen, 1,2,3 P Paul F M Kuijer ${ }^{1,2,3}$
}

\begin{abstract}
- Additional material is published online only. To view please visit the journal online (http://dx.doi.org/10.1136/ oemed-2017-104339).

${ }^{1}$ Coronel Institute of Occupational Health, University of Amsterdam, Academic Medical Center, Amsterdam, The Netherlands

${ }^{2}$ Netherlands Center for Occupational Diseases, Amsterdam, The Netherlands ${ }^{3}$ Amsterdam Public Health research institute, Amsterdam, The Netherlands

${ }^{4}$ School of Occupational Medicine, Department of Medical and Surgical Sciences, University of Bologna, Bologna, Italy
\end{abstract}

\section{Correspondence to}

Dr Henk F van der Molen, Academic Medical Center, Coronel Institute of Occupational Health, PO Box 22660, Amsterdam 1100 DD, The Netherlands; h.f. vandermolen@amc.nl

Received 1 February 2017 Revised 24 May 2017 Accepted 17 June 2017 Published Online First 29 July 2017

\section{ABSTRACT}

The objective of this systematic review and metaanalysis is to examine which work-related risk factors are associated with specific soft tissue shoulder disorders. We searched the electronic databases of Medline and Embase for articles published between 2009 and 24 March 2016 and included the references of a systematic review performed for the period before 2009. Primary cross-sectional and longitudinal studies were included when outcome data were described in terms of clinically assessed soft tissue shoulder disorders and at least two levels of work-related exposure were mentioned (exposed vs less or non-exposed). Two authors independently selected studies, extracted data and assessed study quality. For longitudinal studies, we performed meta-analyses and used GRADE (Grades of Recommendations, Assessment, Development and Evaluation) to assess the evidence for the associations between risk factors and the onset of shoulder disorders. Twenty-seven studies met the inclusion criteria. In total, 16300 patients with specific soft tissue shoulder disorders from a population of 2413722 workers from Denmark, Finland, France, Germany and Poland were included in the meta-analysis of one case-control and six prospective cohort studies. This meta-analysis revealed moderate evidence for associations between shoulder disorders and arm-hand elevation $(\mathrm{OR}=1.9$, $95 \% \mathrm{Cl} 1.47$ to 2.47 ) and shoulder load ( $\mathrm{OR}=2.0$, $95 \% \mathrm{Cl} 1.90$ to 2.10 ) and low to very low evidence for hand force exertion $(\mathrm{OR}=1.5,95 \% \mathrm{Cl} 1.25$ to 1.87), hand-arm vibration $(\mathrm{OR}=1.3,95 \% \mathrm{Cl} 1.01$ to 1.77$)$, psychosocial job demands $(\mathrm{OR}=1.1,95 \% \mathrm{Cl} 1.01$ to 1.25) and working together with temporary workers $(\mathrm{OR}=2.2,95 \% \mathrm{Cl} 1.2$ to 4.2$)$. Low-quality evidence for no associations was found for arm repetition, social support, decision latitude, job control and job security. Moderate evidence was found that arm-hand elevation and shoulder load double the risk of specific shoulder disorders. Low to very-low-quality evidence was found for an association between hand force exertion, handarm vibration, psychosocial job demands and working together with temporary workers and the incidence of specific shoulder disorders.

\section{INTRODUCTION}

Shoulder disorders are frequently reported in the working population, with varying prevalence rates for non-specific shoulder pain (SP) of up to $31 \%$; for clinically assessed specific shoulder disorders such as rotator cuff syndrome (RCS) of up to $6.6 \%$ for men and $8.5 \%$ for women ${ }^{1}$; and for incidences of surgery for subacromial impingement syndrome (SIS) of 11 per 10000 person-years. ${ }^{2}$ Moreover, in numerous countries, work-related shoulder disorders are reported as frequently occurring compensation claims or occupational diseases in various jobs and sectors of industry. ${ }^{3-5}$

Shoulder disorders represent various clinical diagnoses, varying from ICD-10 (International Classification of Diseases) codes M75.0-75.5, that is, adhesive capsulitis (M75.0), RCS—including tendinitis of the supraspinatus, infraspinatus and/or non-traumatic tears and ruptures-(M75.1), bicipital tendinitis (M75.2), calcific tendinitis (M75.3), impingement (M75.4) and bursitis (M75.5), to unspecified soft tissue disorders related to use, overuse and pressure (M70.9). Non-specific SP may be a precursor of specific shoulder disorders, but may also reflect adverse physical, psychological or psychosocial conditions. ${ }^{6}$ For work-related specific shoulder disorders, the biomechanical factors seem to be the most important; however, more recent research also stipulates that psychosocial factors may contribute to proximal factors such as biomechanical constraints. ${ }^{5}$ van Rijn et $a l^{7}$ concluded in their systematic review, based on cross-sectional studies, that highly repetitive work, forceful exertion in work, awkward postures and high psychosocial job demand are associated with the occurrence of SIS.

In primary clinical practice, however, the specific disease classifications of M75.1 through 75.5 are difficult to diagnose and are often assessed with the same medical interview and the physical tests. Moreover, they often present as mixed forms, for example, impingement and bursitis. In the Netherlands, a multidisciplinary guideline of the Dutch Association of Orthopaedics (2012) was recently developed for the diagnosis and treatment of subacromial pain syndrome (SAPS), including the diagnosis of M75.1-75.5, ${ }^{89}$ which is used as clinical outcome in this systematic review.

Knowledge of work-related risk factors associated with SAPS is important in order to initiate primary and secondary preventive interventions at worksites. Previous systematic reviews to determine work-related risk factors for specific soft tissue shoulder disorders ${ }^{7}{ }^{10}$ do not include more recent studies with longitudinal study designs (eg, ref 2) that can assess work-related risk factors for the onset of specific shoulder disorders. This systematic review aimed to examine: (i) which work-related risk factors contribute to the onset of clinically assessed SAPS and (ii) to what extent these risk factors are associated with clinically assessed SAPS. 


\section{METHODS}

\section{Protocol and registration}

This review followed the PRISMA (Preferred Reporting Items for Systematic Reviews and Meta-Analyses) statement and the study protocol was registered at PROSPERO (ID= CRD42016039059), http://www.crd.york.ac.uk/PROSPERO.

\section{Study selection}

\section{Eligibility criteria}

Primary cross-sectional, case-control and cohort studies were included when outcome data were described in terms of clinically assessed soft tissue shoulder disorder (present or not) and at least two levels of work-related exposure (exposed vs less or non-exposed) among a working population were reported in order to be able to retrieve or calculate a risk estimate.

Based on van Rijn et al, ${ }^{7}$ we defined six types of exposure: (1) force, (2) posture, (3) movement, (4) hand-arm vibration, (5) shoulder load as combined exposure measure and (6) psychosocial risk factors. Clinically assessed soft tissue shoulder disorders were grouped into SAPS, defined as all non-traumatic, usually unilateral, shoulder problems that cause pain, localised around the acromion, often worsening during or subsequent to lifting of the arm. The different clinical and/or radiological names, such as bursitis, tendinosis calcarea, supraspinatus tendinopathy, partial tear of the rotator cuff, biceps tendinitis (BT) or tendon cuff degeneration, are all part of SAPS. ${ }^{89}$

All types of clinical assessments were eligible for inclusion, such as work anamnesis, physical tests, questionnaires on localised pain and imaging. Studies that described work-related risk factors in terms of job or occupation, physical workload, specific occupational activities such as repetitive arm movements, or postures such as arm elevation were eligible for inclusion. The studies had to describe workers in a real workplace setting and therefore no experimental studies were included. All types of exposure assessment were eligible for inclusion: self-reports, researcher observations or direct measurements. No additional criteria were formulated regarding latency between exposure and the presence or onset of the disorder or adjustment for confounders. The exclusion criteria concerning the outcome of clinically assessed soft tissue shoulder disorders were: nerve compressions, radiating pain from cervical spine, osteoarthritis and systemic diseases.

\section{Data sources and search terms}

We searched the electronic databases of Medline and Embase for studies between 2009 and 24 March 2016 as described in online supplementary appendix 1 . Our PICO can be stated as: $\mathrm{P}=$ working population, $\mathrm{I} / \mathrm{C}$ exposed/less or none exposed to a priori defined exposure categories, $\mathrm{O}=\mathrm{SAPS}$. To construct the search strategy (JGD, HFM) a reference set of 23 potentially eligible studies was collected. Of these, 17 studies were derived from the systematic review by van Rijn et al, 3 additional studies from a citation check of all these studies in Google Scholar and another 3 additional studies from an orienting (scoping) search. All 23 studies had to be retrieved to validate applied terms and concepts of the search strategy. Eligible studies before 2009 were retrieved from the systematic review by van Rijn et al. ${ }^{7}$

\section{Data collection and analyses}

\section{Study selection process}

Titles and abstracts were independently screened by two review authors (HFM, CF or PPFMK) to identify potentially relevant studies. We used an online software tool to screen and assess references (https://www.covidence.org). The full texts of potentially relevant articles were assessed for eligibility against the inclusion criteria. Disagreement between review authors on the selection of studies for inclusion occurred in relation to about $5 \%$ of the references screened and was resolved by discussion.

\section{Data extraction and management}

Data were extracted by two review authors (HFM and CF) and checked by another review author (PPFMK). Data on the following were extracted from each article: author; country of study; study design (cohort, case-control or cross-sectional study); case definition of specific shoulder disorder; sources and number of participants; exposure definition; exposure assessment; exposure categories; risk estimate and adjustment for confounders.

\section{Methodological quality assessment}

Methodological quality was assessed for the studies that reported on risk factors; studies that reported on job title were analysed descriptively without quality assessment. The quality of the studies was independently assessed by two review authors (HFM, PPFMK or CF). For all study designs, the slightly adapted quality criteria (see online supplementary appendix 2) from the systematic review by van Rijn et $a l^{7}$ were used. The quality criteria for exposure definition and assessment were reformulated into: (1) at least two aspects of duration, frequency and intensity of exposure; and (2) $\geq 3$ exposure categories reported (in order to detect a dose-response).

In total, 16 items across five categories for quality assessment were assessed (see also online supplementary appendix 2): (1) study population, (2) assessment exposure,(3) assessment outcome, (4) study design and (5) data analysis. The criteria for each item were scored with 'positive', 'negative' or 'not clear'. There was disagreement about 19 out of 240 items, all of which were resolved by discussion. High quality was defined as $\geq 11$ items scored as 'positive' out of 16 quality criteria.

\section{Association measures between work-related risk factors or job title and SAPS}

Risk estimates and the corresponding 95\% CIs of the association between work-related factors and SAPS were extracted or calculated and summarised. Risk estimates concerning the association between job title and shoulder disorders were described and summarised. Three review authors (HFM, CF, PFMK) discussed and decided on the risk estimates to be included in the meta-analysis.

\section{Data synthesis}

A descriptive analysis of all studies was performed, summarised, classified into categories of physical and psychosocial risk factors, and assessed for methodological quality.

\section{Meta-analyses and quality of evidence}

The selection of the work-related risk factors in the meta-analyses was based on: (1) sufficient contrast between reported exposure categories, that is, low versus high exposure; (2) clearly defined exposure criteria suitable for exposure assessment at worksites; and (3) effect estimates controlled for other non-work-related factors, as reported in the primary studies. Risk estimates for 
both men and women were selected when the study provided only sex-specific estimates.

The meta-analysis was performed in line with the procedure described in Watanabe et al. ${ }^{11}$ : all risk estimates were transformed into a natural logarithm. The SE for a log-transformed OR was calculated based on the $95 \%$ CI for the risk estimate. A log-transformed OR and its SE were used for the meta-analysis in Review Manager (Cochrane Review Manager V.5.3). For the main analysis, the main ORs and the SEs from selected studies were subjected to a random-effects model meta-analysis to estimate a pooled OR and its $95 \% \mathrm{CI}$.

The quality of evidence was assessed using the GRADE (Grades of Recommendations, Assessment, Development and Evaluation) framework for prognostic studies developed by Huguet et al. ${ }^{12}$ The starting point for the quality of the evidence was 'high' for longitudinal studies that sought to confirm independent associations between the prognostic factor and the outcome ('Phase 2' explanatory studies). The evidence could decrease on the basis of five factors: study limitations, inconsistency, indirectness, imprecision and publication bias. Moreover, two factors: (1) study findings with moderate or large effect sizes (ie, lower limit of 95\% CI, OR>2.0) or (2) an exposure-response gradient, could lead to an upgrade of the quality of evidence. Four levels of quality were used: high, moderate, low and very low.

\section{RESULTS}

\section{Selected studies}

A PRISMA flow diagram of the study selection process is shown in figure 1. After excluding duplicates, 2744 references were retrieved from the databases and the systematic review by van Rijn et $a l^{7}$ and assessed based on title and abstract. The full texts of 72 potentially eligible articles were then examined, of which 31 articles met the inclusion criteria, 4 of which contained the same study population and outcome of interest, resulting in 27 articles included in this review. Of the 27 articles, 12 described job title and sectors of industry, 13 described risk factors and 2 described both. Six diagnoses were studied: RCS or rotator cuff tendinitis, infraspinatus tendinitis, supraspinatus tendinitis, SIS, BT, SP with clinical test, all part of the SAPS case definition.

\section{Job title and SAPS}

Fourteen studies ${ }^{13-26}$ described the association between performing a specific job or working in a sector of industry and the occurrence of clinically assessed SAPS (including the diagnoses M75.1-75.5) (see online supplementary appendix 3). The following jobs and sectors had an increased risk of SAPS: assembly workers, ${ }^{14}$ fish processing workers, ${ }^{15}$ slaughterhouse workers, ${ }^{16}$ sewing machine operators, ${ }^{17}$ manual workers, ${ }^{19}$ fishermen, ${ }^{20}$

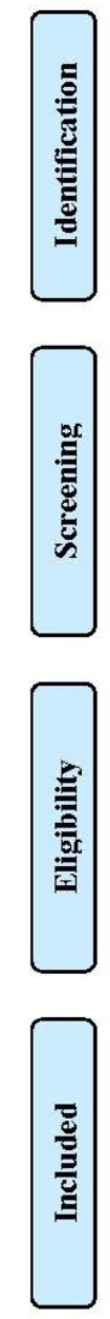

Records identified through database searching 2009 - March 2016 $(\mathrm{n}=3452)$
Records identified through references of systematic review Van Rijn et al. (2010) $(\mathrm{n}=17)$

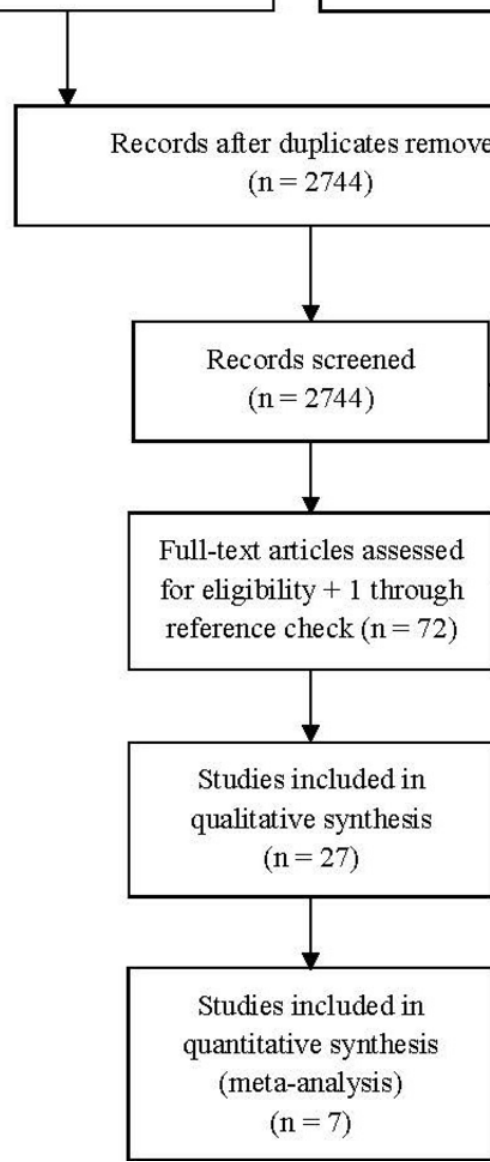

2673 records excluded on title and abstract

Figure 1 PRISMA (Preferred Reporting Items for Systematic Reviews and Meta-Analyses) flow diagram. 
construction and interior workers, ${ }^{21}$ metal workers, ${ }^{21}$ nurses, ${ }^{24}$ and workers in the army, air force and marines. ${ }^{25}$

\section{Risk factors and SAPS}

In total, seven longitudinal studies ${ }^{2127-31}$ and eight cross-sectional studies ${ }^{622} 32-37$ described the association between exposure to physical and psychosocial risk factors and the occurrence of clinically assessed SAPS (see online supplementary appendix 4). The characteristics of the outcome definition (SAPS) and exposure definition (in intensity, frequency and duration) are presented in table 1 .

\section{Methodological quality}

The methodological quality of the studies of risk factors varied from scoring 7 out of 16 items to 16 out of 16 items (see table 2). The most frequently missing quality items were the lack of blinding for outcome status (ie, specific shoulder disorder) and the reverse, the lack of blinding for exposure status, as well as a lack of information about completers versus withdrawals from the study. All six cohort and one case-control studies met the quality level of $\geq 11$ out of 16 quality criteria; among the eight cross-sectional studies five met $\geq 11$ quality criteria.

\section{Meta-analyses and assessment of evidence}

In total, 16300 patients with specific shoulder disorders from a population of 2413722 workers from Denmark, Finland, France, Germany and Poland were used in the meta-analysis. Two study populations were overlapping to some extent, ${ }^{2} 31$ therefore the risk estimates in the cohort study of Svendsen et $a l^{31}$ were censored by the original authors for the period from 1996 through 2002. Figure 2 and additional figures in online supplementary material summarise the results of the meta-analyses, while table 3 summarises the assessment of evidence concerning risk factors for specific shoulder disorders.

\section{Arm elevation}

Three cohort studies 2831 and one case-control study ${ }^{21}$ demonstrate that there is moderate quality evidence that arm elevation increases the incidence of SAPS, with a pooled OR of $1.91(95 \%$ CI 1.47 to 2.47) (table 3), comparable to the pooled OR of 2.12 (95\% CI 1.74 to 2.58 ) (figure $2 \mathrm{~A}$ ) when also including the cross-sectional studies $(n=5)$ of sufficient quality.

\section{Shoulder load}

Two cohort studies ${ }^{231}$ demonstrate that there is moderate quality evidence that shoulder load increases the incidence of SAPS, with a pooled OR of 2.00 (95\% CI 1.90 to 2.10) (table 3 and figure $2 \mathrm{~B}$ ).

\section{Hand-arm force exertion}

Four cohort studies 282931 and one case-control study ${ }^{21}$ demonstrate that there is low-quality evidence that hand-arm force exertion increases the incidence of SAPS, with a pooled OR of 1.53 (95\% CI 1.25 to 1.87 ) (table 3), comparable to the pooled OR of 1.56 (95\% CI 1.26 to 1.93) (online supplementary figure 2.3) when also including the cross-sectional studies $(n=3)$ of sufficient quality.

\section{Arm-hand repetition}

Three cohort studies ${ }^{29} 31$ demonstrate that there is low-quality evidence for no increased incidence of SAPS due to arm-hand repetition with a pooled OR of 1.42 (95\% CI 0.91 to 2.22) (table 3), which is significantly lower compared with the pooled
OR of 1.70 (95\% CI 1.18 to 2.46) (online supplementary figure 2.4) when also including the cross-sectional studies $(n=2)$ of sufficient quality.

\section{Hand-arm vibration}

Three cohort studies ${ }^{2729}$ and one case-control study ${ }^{21}$ demonstrate that there is low-quality evidence that hand-arm vibration increases the incidence of SAPS, with a pooled OR of 1.34 (95\% CI 1.01 to 1.77 ) (table 3), comparable to the pooled OR of 1.34 (95\% CI 1.02 to 1.76 ) (online supplementary figure 2.5) when also including the cross-sectional study $(n=1)$ of sufficient quality.

\section{Psychosocial demands}

Three cohort studies ${ }^{29-31}$ demonstrate that there is low-quality evidence that psychosocial demands increase the incidence of SAPS, with a pooled OR of 1.12 (95\% CI 1.01 to 1.25 ) (table 3 ), comparable to the pooled OR of 1.14 (95\% CI 1.04 to 1.24 ) (online supplementary figure 2.6) when also including the cross-sectional studies $(n=3)$ of sufficient quality.

\section{Social support}

Three cohort studies ${ }^{283031}$ demonstrate that there is low-quality evidence for no increased incidence of SAPS due to low social support from colleagues and/or manager, with a pooled OR of 1.05 (95\% CI 0.83 to 1.33 ) (table 3), comparable to the pooled OR of 1.02 (95\% CI 0.84 to 1.24) (online supplementary figure 2.7) when also including the cross-sectional study $(n=1)$ of sufficient quality.

\section{Decision latitude}

Two cohort studies ${ }^{29} 30$ demonstrate that there is low-quality evidence for no increased incidence of SAPS due to low decision latitude, with a pooled OR of 1.08 (95\% CI 0.89 to 1.31$)$ (table 3), which is comparable to the pooled OR of 1.01 (95\% CI 0.81 to 1.25 ) (online supplementary figure 2.8) when also including the cross-sectional study $(n=1)$ of sufficient quality.

\section{Job control, job security and working with temporary workers}

One cohort study ${ }^{31}$ demonstrates that there is low-quality evidence for no increased incidence of SAPS due to low job control, with an OR of 1.22 (95\% CI 1.00 to 1.50). One cohort study ${ }^{30}$ demonstrated that there is low-quality evidence for no increased incidence of SAPS due to low job security, with an OR of 1.12 (95\% CI 0.93 to 1.36). One cohort study ${ }^{28}$ demonstrated very-low-quality evidence that for female workers there is an increased incidence of SAPS when working together with temporary workers, with an OR of 2.2 (95\% CI 1.2 to 4.2) (table 3).

\section{DISCUSSION \\ Main findings}

This systematic review, including a meta-analysis, revealed moderate evidence that arm elevation and shoulder load increase the incidence of specific shoulder disorders, with estimated pooled ORs around 2.0. Low to very-low-quality evidence was found for an association between hand force exertion, hand-arm vibration, psychosocial job demands and working together with temporary workers and the incidence of specific shoulder disorders. Low-quality evidence suggesting no associations was found for repetitive arm movements, social support, decision latitude, job control and job security.

Most longitudinal studies were controlled for the personal factors of age and sex, for non-work-related factors such as 


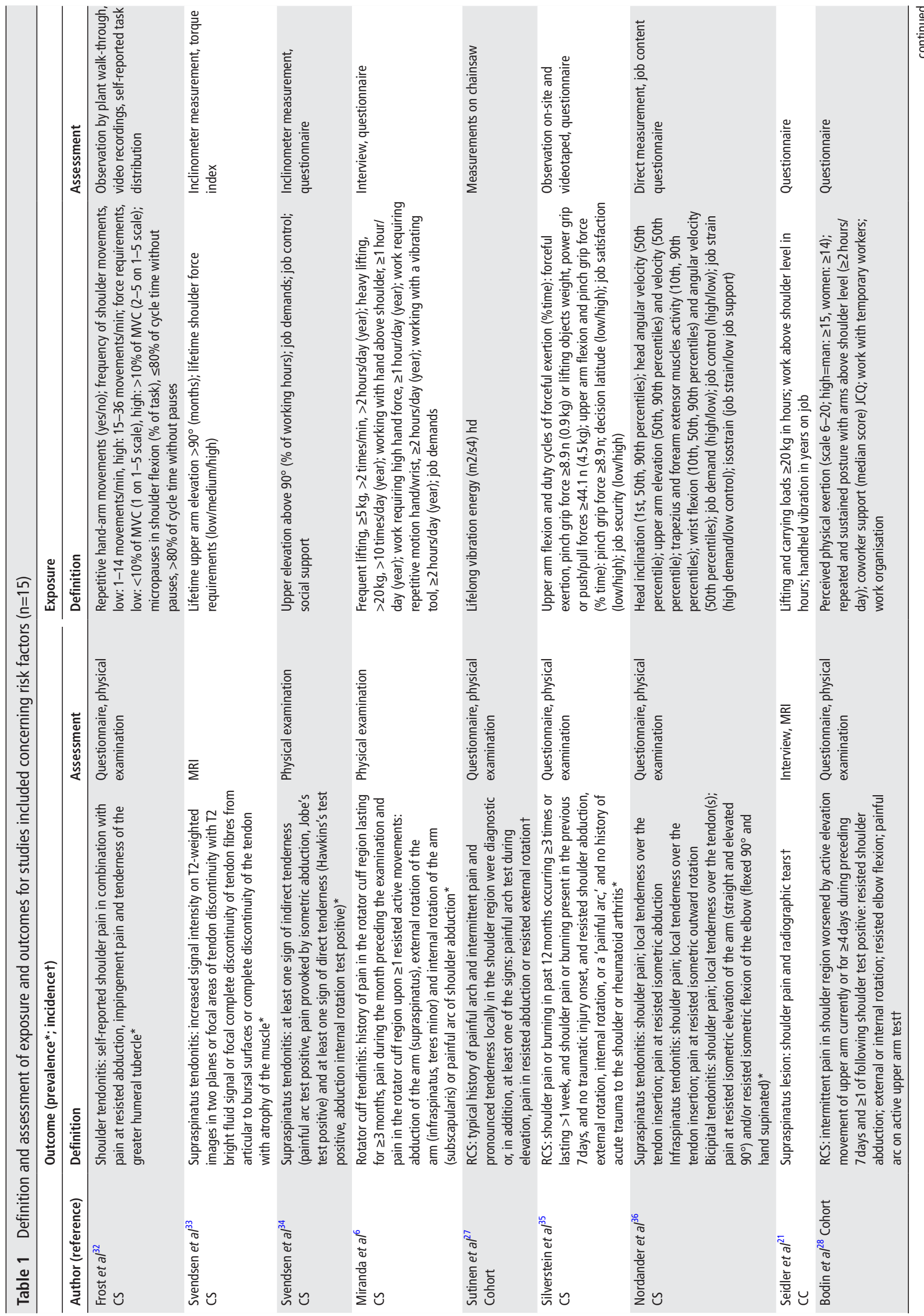

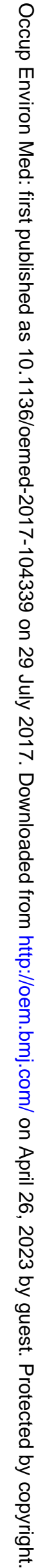




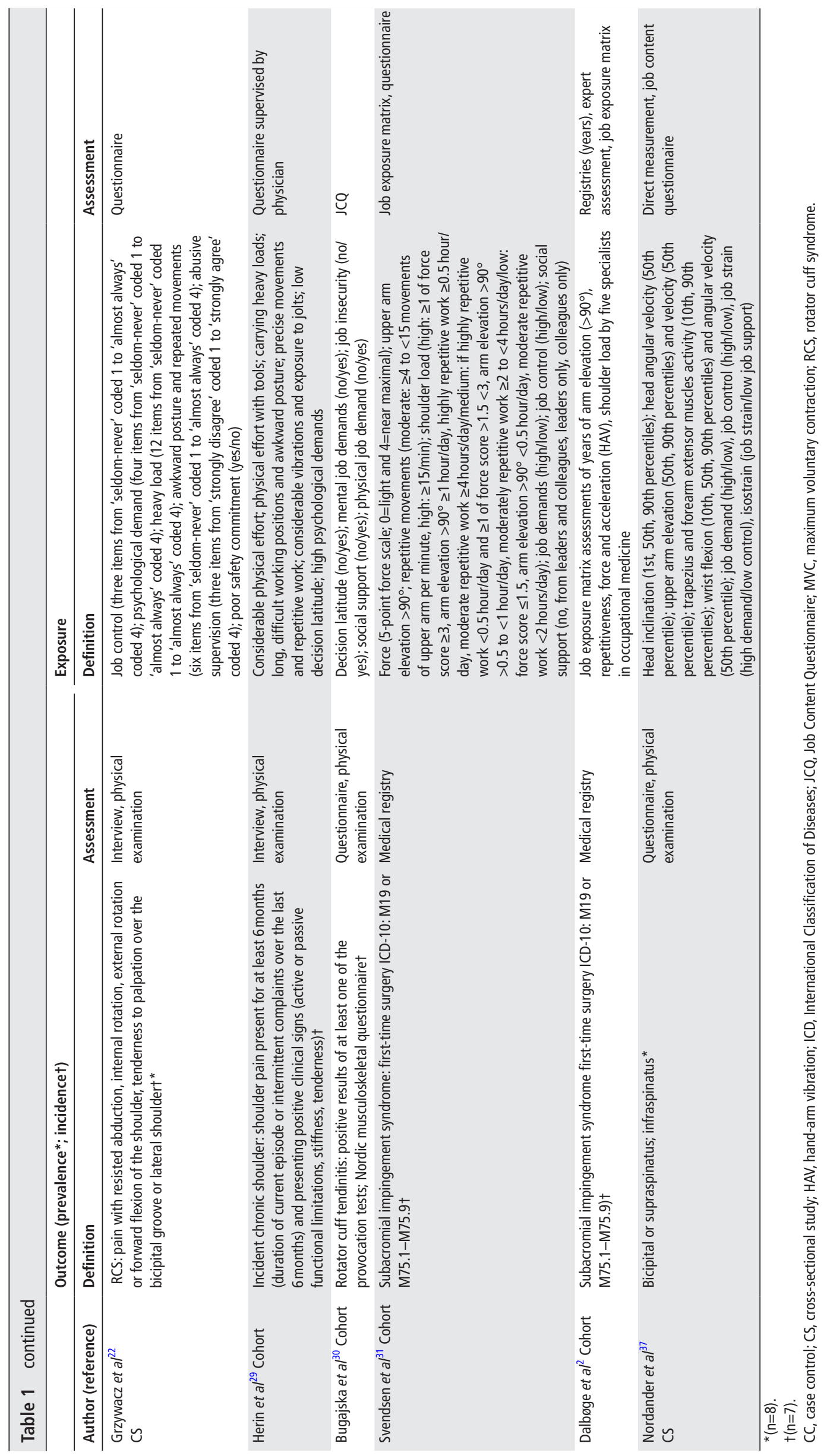




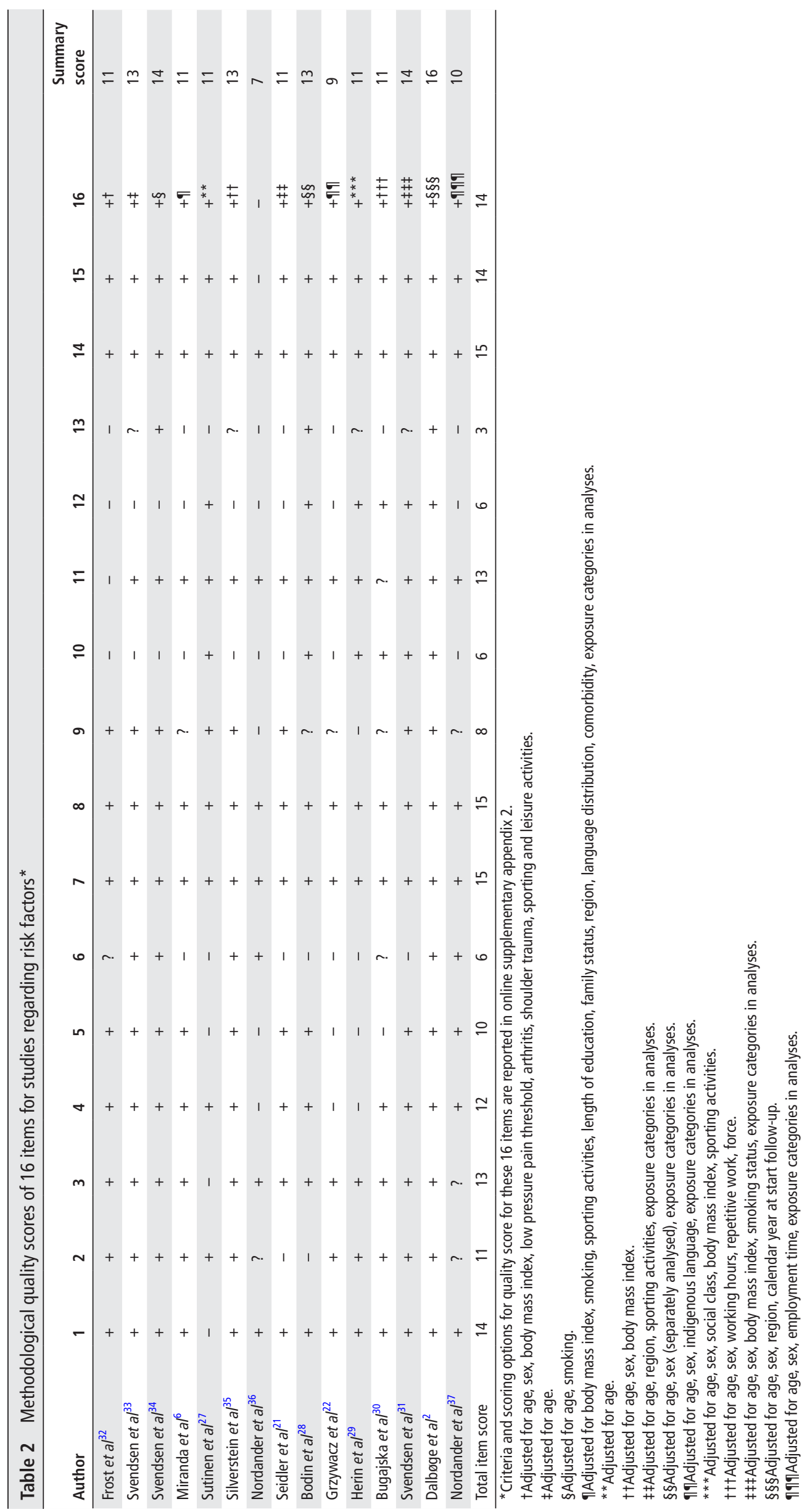


A

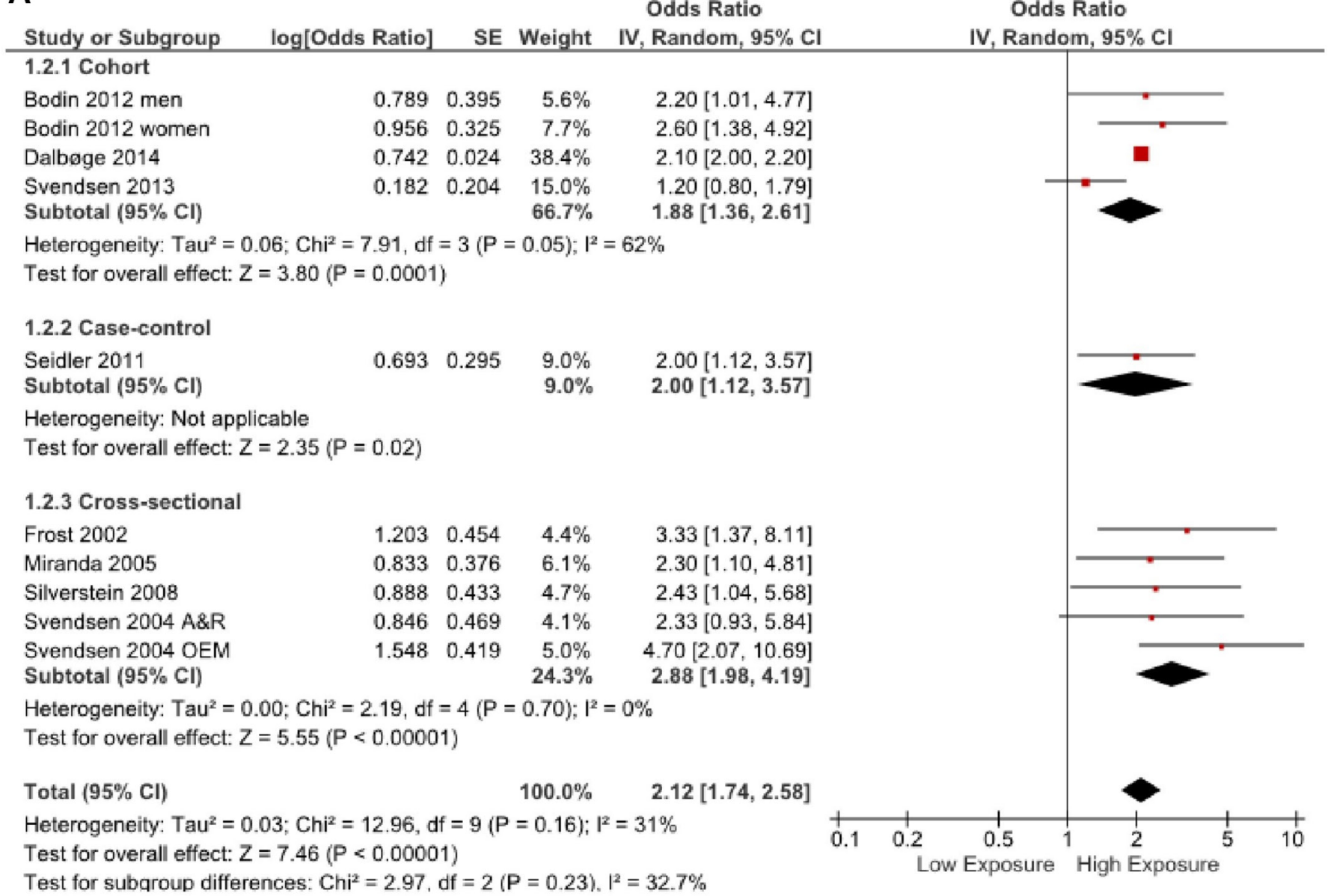

B

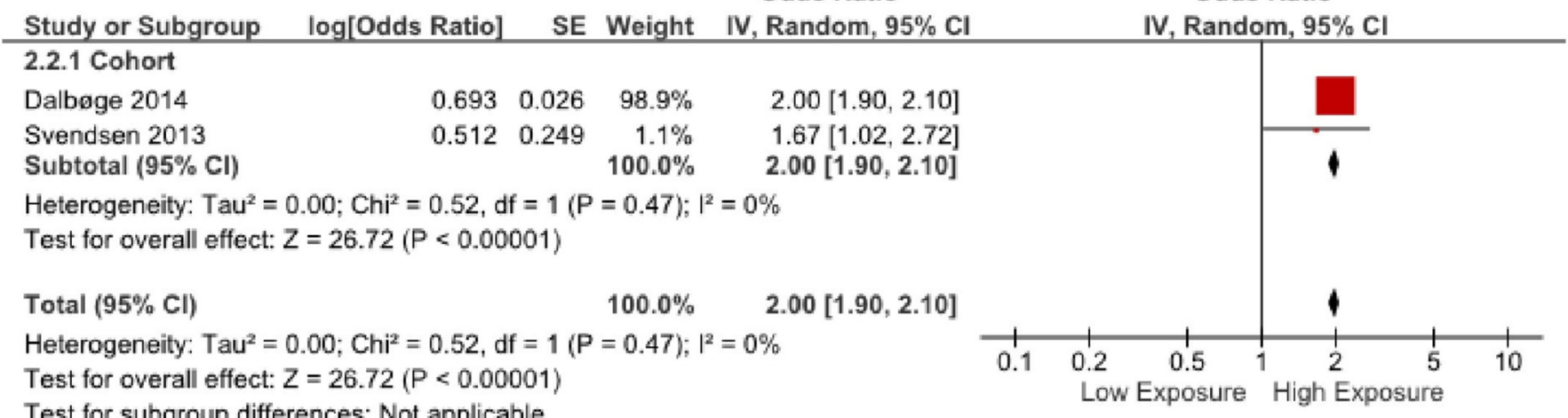

Figure 2 Forest plots. (A) Forest plot of studies regarding arm elevation and SAPS. (B) Forest plot of studies regarding shoulder load and SAPS. df, degrees of freedom; SAPS, subacromial pain syndrome.

specific leisure time or sport activities, and for other work-related factors. This could be a reason why the psychosocial factors were found to contribute little to the incidence of these specific shoulder disorders. The biological pathway for specific shoulder disorders seems to be biomechanical in nature (eg, ref ${ }^{38}$ ), while psychosocial factors might act as more intermediating factors 5 influencing these biomechanical factors.

This review confirmed and strengthened the evidence on risk factors as reported in the systematic review by van Rijn et al. ${ }^{7}$ Based on the more recent published prospective cohort and case-control studies used in our review, the most important risk factors for clinically assessed soft tissue-specific shoulder disorders defined as SAPS are arm elevation and shoulder load in terms of combined physical exposures. Psychosocial factors seem to contribute to a much lesser extent in the onset of specific shoulder disorders.

\section{Risk factors and SAPS}

The exposure categories for the risk factors and the case definitions of the specific shoulder disorders varied in the included studies. Therefore, we have explicitly described all definitions 


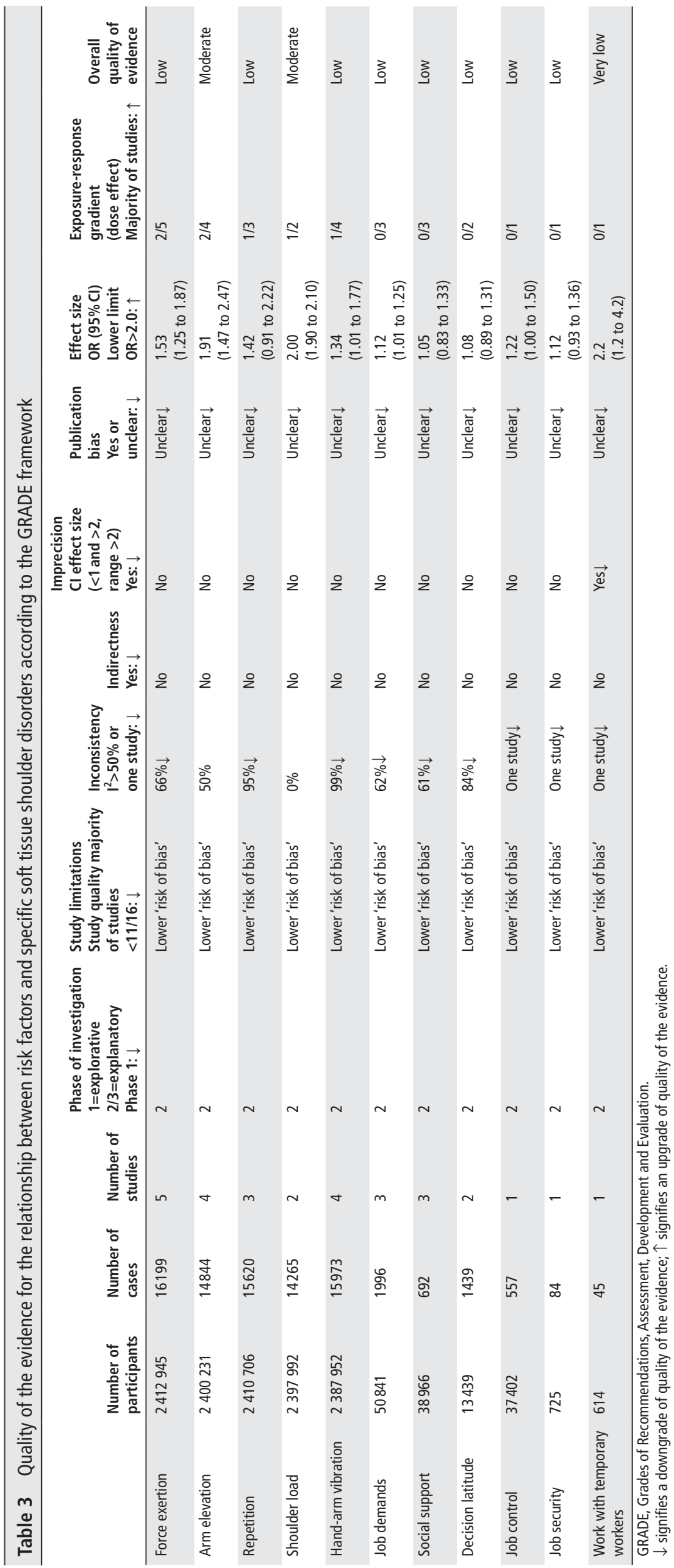


and assessments (see table 1) and reported outcomes (see online supplementary appendix 4). The variability in exposure categories of the retrieved risk factors, however, did not allow to combine comparable exposure levels across studies into more detail.

The variability in case definitions of specific shoulder disorders was partly due to the different clinical settings, which varied from a primary care setting to a clinical specialist. Although case definitions for the specific shoulder disorders varied, all studies in the formal and secondary meta-analyses clinically assessed these disorders using a combination of symptoms and signs in terms of diagnostic physical testing and/or imaging as recommended in the multidisciplinary guideline for diagnosis of SAPS. ${ }^{8}{ }^{9}$ This guideline also proposes using a combination of clinical tests to increase post-test probability of a diagnosis of SAPS. Consequently, further research in the area of clinical assessment of specific shoulder disorders (eg, ${ }^{39}{ }^{40}$ combined with more detailed exposure assessment in using intensity, duration and frequency of risk factors $\left(\mathrm{eg}, \mathrm{ref}^{2}\right.$ ) will contribute to a better insight into the pathogenic pathways and attributional fractions of worksite exposure.

\section{Methodological considerations}

Meta-analyses were performed to estimate pooled associations, although there was heterogeneity in the clinically assessed shoulder disorders and the assessment of exposure to risk factors in the longitudinal studies included in this review. For each risk factor we have chosen to contrast between the lowest and highest exposure category for answering our research questions whether or not an increased risk is present and to what extent. To prevent data-driven approaches, we did not select other exposures between low and high. Furthermore, a reported dose-response relation in the original studies was used in the GRADE assessment. For specific shoulder disorders we used the case definition of SAPS, although in two studies ${ }^{21}$ it was not possible to differentiate the results between SAPS diagnoses and a supposed small proportion of osteoarthritis cases.

Heterogeneity, however, was explicitly addressed through the grading of the quality of evidence. Publication bias was difficult to assess because of the small number of studies included in each risk factor. Taking cross-sectional studies into accountalso including smaller study groups with and without statistically significant associations-made little difference to the estimates of the pooled ORs, with exception of repetitive arm movements. However, we treated the assessment of publication bias conservatively by downgrading the evidence in the case of lack of clarity.

In this systematic review based on a validated search in two databases, all three criteria for the establishment of work-related disorders ${ }^{41}$ were encountered, namely clinical diagnosis, exposure and evidence for causal relationships. Evidence for causal relationships was based on the meta-analyses and grading of the evidence. The choice of risk factors for the meta-analyses was transparent, and based on maximal contrast between exposure categories and feasibility of assessment for diagnosis and exposure assessment in general practice. Exploration of the formal meta-analyses (prospective cohort and case-control studies) by including cross-sectional studies yielded associations in the same direction.

\section{Prevention}

Knowledge of evidence-based work-related risk factors can stimulate primary and secondary prevention. Preventive actions to reduce arm elevation and shoulder load as combined physical exposures, for example, hand force exertion and arm elevation, are recommended to prevent specific work-related shoulder disorders. Psychosocial demands and working together with colleagues in temporary employment were the only psychosocial factors that revealed significant associations with specific shoulder disorders in this review. It is possible that psychosocial factors play an intermediate role, ${ }^{5} 38$ and therefore should also be targeted in occupational preventive actions. In addition, as psychosocial factors play a more prominent role in the onset and prevalence of non-specific SPthrough other biological pathways such as muscle strain from the neck and shoulder muscles-targeted interventions counteracting these factors might also be considered. There are indications that specific strength training ${ }^{42-44}$ or just general exercise training, ${ }^{45}$ adjustment to furniture and equipment ${ }^{46}$ and improving work techniques like taking more breaks and reducing work demands can reduce specific and non-specific shoulder disorders. ${ }^{47}$

\section{CONCLUSIONS}

Arm-hand elevation and shoulder load double the risk of specific soft tissue shoulder disorders. Low to very-low-quality evidence was found for an association between hand force exertion, hand-arm vibration, psychosocial job demands and working together with temporary workers and the incidence of specific shoulder disorders.

Acknowledgements We are very grateful to the authors of the paper by Svendsen et $a^{\beta^{1}}$ for the reanalyses of their data so we could incorporate their outcomes in our meta-analyses.

Contributors HFM initiated the study, performed the statistical analyses and drafted the manuscript. JGD contributed to the literature search. HFM, CF and PFMK performed the study selection, data extraction and interpretation of the data. MHWFD was the principle investigator in this study. All authors made substantial contributions to the conception of the study and manuscript.

Funding The work was sponsored by the Ministry of Social Affairs and Employment, the Netherlands (Grant No. 5100-22709).

Competing interests None declared.

Provenance and peer review Not commissioned; externally peer reviewed.

(C) Article author(s) (or their employer(s) unless otherwise stated in the text of the article) 2017. All rights reserved. No commercial use is permitted unless otherwise expressly granted.

\section{REFERENCES}

1 Bodin J, Ha C, Chastang JF, et al. Comparison of risk factors for shoulder pain and rotator cuff syndrome in the working population. Am J Ind Med 2012;55:605-15.

2 Dalbøge A, Frost P, Andersen JH, et al. Cumulative occupational shoulder exposures and surgery for subacromial impingement syndrome: a nationwide Danish cohort study. Occup Environ Med 2014;71:750-6.

3 Beach J, Senthilselvan A, Cherry N. Factors affecting work-related shoulder pain. Occup Med 2012:62:451-4.

4 van der Molen HF, de Vries SC, Stocks SJ, et al. Incidence rates of occupational diseases in the dutch construction sector, 2010-2014. Occup Environ Med 2016;73.

5 Bodin J, Garlantézec R, Costet N, et al. Forms of work organization and associations with shoulder disorders: results from a french working population. Appl Ergon 2017;59:1-10.

6 Miranda H, Viikari-Juntura E, Heistaro S, et al. A population study on differences in the determinants of a specific shoulder disorder versus nonspecific shoulder pain without clinical findings. Am J Epidemio/ 2005;161:847-55.

7 van Rijn RM, Huisstede BM, Koes BW, et al. Associations between work-related factors and specific disorders of the shoulder--a systematic review of the literature. Scand J Work Environ Health 2010;36:189-201.

8 Guideline Dutch Association of Orthopedics. Richtlijn diagnostiek en behandeling van het subacromiaal pijnsyndroom. 2012. http://www.orthopeden.org/ (accessed 26 Dec 2016).

9 Diercks R, Bron C, Dorrestijn O, et al. Guideline for diagnosis and treatment of subacromial pain syndrome: a multidisciplinary review by the dutch Orthopaedic Association. Acta Orthop 2014;85:314-22. 
10 Sluiter JK, Rest KM, Frings-Dresen MH. Criteria document for evaluating the workrelatedness of upper-extremity musculoskeletal disorders. Scand J Work Environ Health 2001;27 Suppl 1:1-102.

11 Watanabe K, Imamura K, Kawakami N. Working hours and the onset of depressive disorder: a systematic review and meta-analysis. Occup Environ Med 2016;73.

12 Huguet A, Hayden JA, Stinson J, et al. Judging the quality of evidence in reviews of prognostic factor research: adapting the GRADE framework. Syst Rev 2013;2:71.

13 Luopajärvi T, Kuorinka I, Virolainen M, et al. Scand J Work Environ Health 1979;5:48-55.

14 Park RM, Nelson NA, Silverstein MA, et al. Use of medical insurance claims for surveillance of occupational disease. An analysis of cumulative trauma in the auto industry. J Occup Med 1992;34:731-7.

15 Nordander C, Ohlsson K, Balogh I, et al. Fish processing work: the impact of two sex dependent exposure profiles on musculoskeletal health. Occup Environ Med 1999;56:256-64.

16 Frost $\mathrm{P}$, Andersen JH. Shoulder impingement syndrome in relation to shoulder intensive work. Occup Environ Med 1999;56:494-8.

17 Kaergaard A, Andersen JH. Musculoskeletal disorders of the neck and shoulders in female sewing machine operators: prevalence, incidence, and prognosis. Occup Environ Med 2000;57:528-34.

18 Wang LY, Pong YP, Wang HC, et al. Cumulative trauma disorders in betel pepper leaf-cullers visiting a rehabilitation clinic: experience in Taitung. Chang Gung Med J 2005;28:237-46.

19 Melchior M, Roquelaure Y, Evanoff B, et al. Why are manual workers at high risk of upper limb disorders? the role of physical work factors in a random sample of workers in France (the Pays de la Loire study). Occup Environ Med 2006;63:754-61.

20 Kaerlev L, Jensen A, Nielsen PS, et al. Hospital contacts for injuries and musculoskeletal diseases among seamen and fishermen: a population-based cohort study. BMC Musculoskelet Disord 2008;9:8.

21 Seidler A, Bolm-Audorff U, Petereit-Haack G, et al. Work-related lesions of the supraspinatus tendon: a case-control study. Int Arch Occup Environ Health 2011;84:425-33.

22 Grzywacz JG, Arcury TA, Mora D, et al. Work organization and musculoskeletal health: clinical findings from immigrant latino poultry processing and other manual workers. Occup Environ Med 2012;54:995-1001.

23 Rosenbaum DA, Grzywacz JG, Chen H, et al. Prevalence of Epicondylitis, rotator cuff syndrome, and low back pain in Latino poultry workers and manual laborers. Am J Ind Med 2013;56:226-34.

24 Chung YC, Hung CT, Li SF, Sf L, et al. Risk of musculoskeletal disorder among taiwanese nurses cohort: a nationwide population-based study. BMC Musculoskelet Disord 2013;14:144.

25 Hsiao MS, Cameron KL, Tucker CJ, et al. Shoulder impingement in the United States military. J Shoulder Elbow Surg 2015;24:1486-92.

26 Sansone VC, Meroni R, Boria P, et al. Are occupational repetitive movements of the upper arm associated with rotator cuff calcific tendinopathies? Rheumatol Int 2015:35:273-80.

27 Sutinen P, Toppila E, Starck J, et al. Hand-arm vibration syndrome with use of antivibration chain saws: 19 -year follow-up study of forestry workers. Int Arch Occup Environ Health 2006;79:665-71.

28 Bodin J, Ha C, Petit Le Manac'h A, et al. Risk factors for incidence of rotator cuff syndrome in a large working population. Scand I Work Environ Health 2012;38:436-46.
29 Herin F, Vézina M, Thaon I, et al. Predictors of chronic shoulder pain after 5 years in a working population. Pain 2012;153:2253-9.

30 Bugajska J, Zołnierczyk-Zreda D, Jędryka-Góral A, et al. Psychological factors at work and musculoskeletal disorders: a one year prospective study. Rheumatol Int 2013;33:2975-83.

31 Svendsen SW, Dalbøge A, Andersen JH, et al. Risk of surgery for subacromial impingement syndrome in relation to neck-shoulder complaints and occupational biomechanical exposures: a longitudinal study. Scand J Work Environ Health 2013;39:568-77.

32 Frost $\mathrm{P}$, Bonde JPE, Mikkelsen S, et al. Risk of shoulder tendinitis in relation to shoulder. risk of shoulder tendinitis in relation to shoulder loads in monotonous repetitive work. Am J Indust Med 2002;41:11-18.

33 Svendsen SW, Gelineck J, Mathiassen SE, et al. Work above shoulder level and degenerative alterations of the rotator cuff tendons: a magnetic resonance imaging study. Arthritis Rheum 2004;50:3314-22.

34 Svendsen SW, Bonde JP, Mathiassen SE, et al. Work related shoulder disorders: quantitative exposure-response relations with reference to arm posture. Occup Environ Med 2004;61:844-53.

35 Silverstein BA, Bao SS, Fan ZJ, et al. Rotator cuff syndrome: personal, work-related psychosocial and physical load factors. J Occup Environ Med 2008;50:1062-76.

36 Nordander C, Ohlsson K, Akesson I, et al. Risk of musculoskeletal disorders among females and males in repetitive/constrained work. Ergonomics 2009;52:1226-39.

37 Nordander C, Hansson G̊, Ohlsson K, et al. Exposure-response relationships for work-related neck and shoulder musculoskeletal disorders--analyses of pooled uniform data sets. App/ Ergon 2016;55:70-84.

38 Roquelaure Y, Bodin J, Ha C, et al. Personal, biomechanical, and psychosocial risk factors for rotator cuff syndrome in a working population. Scand J Work Environ Health 2011:37:502-11.

39 Hermans J, Luime JJ, Meuffels DE, et al. Does this patient with shoulder pain have rotator cuff disease?: the rational clinical examination systematic review. JAMA 2013;310:837-47.

40 Gismervik SØ, Drogset JO, Granviken F, et al. Physical examination tests of the shoulder: a systematic review and meta-analysis of diagnostic test performance. $B M C$ Musculoskelet Disord 2017;18:41.

41 Verbeek J. When Work is Related to Disease, What Establishes Evidence for a Causal Relation?

42 Pedersen MT, Andersen $\mathrm{CH}$, Zebis MK, et al. Implementation of specific strength training among industrial laboratory technicians: long-term effects on back, neck and upper extremity pain. BMC Musculoskelet Disord 2013;14:287

43 Mortensen P, Larsen Al, Zebis MK, et al. Lasting effects of workplace strength training for neck/shoulder/arm pain among laboratory technicians: natural experiment with 3-year follow-up. Biomed Res Int 2014;2014-.

44 Van Eerd D, Munhall C, Irvin E, et al. Effectiveness of workplace interventions in the prevention of upper extremity musculoskeletal disorders and symptoms: an update of the evidence. Occup Environ Med 2016;73:62-70.

45 Shire AR, Stæhr TAB, Overby JB, et al. Specific or general exercise strategy for subacromial impingement syndrome-does it matter? A systematic literature review and Meta analysis. BMC Musculoskelet Disord 2017:18:158.

46 Ketola $R$, Toivonen $R$, Häkkänen $M$, et al. Effects of ergonomic intervention in work with video display units. Scand J Work Environ Health 2002;28:18-24.

47 Veiersted KB, Gould KS, Osterås N, et al. Effect of an intervention addressing working technique on the biomechanical load of the neck and shoulders among hairdressers. Appl Ergon 2008;39:183-90. 